\title{
In-process feedback control of tube hydro-forming process
}

\author{
Benny Endelt ${ }^{1} \mathbb{D}$
}

Received: 26 August 2021 / Accepted: 4 January 2022 / Published online: 27 January 2022

(c) The Author(s) 2022

\begin{abstract}
The present work will address the problem of designing an in-process control system for a tube hydro-forming process, controlling the tool filling forming a T-tube. The control problem is nontrivial as wrinkling and bursting will develop rapidly and, in most cases, are irreversible - thus, the control system must react fast and without extensive overshoot. The objective is to control the tool filling, reproducing a reference filling trajectory, where the controller input is defined as a correction of the reference forming pressure. The control system was verified experimentally, using four different error scenarios. Initially, the error was provoked, by manipulating the input signal, and for all three cases, the control system successfully eliminates both wrinkling and bursting. Finally, the material was changed going from an aluminum grade 5049-0 to 6060-T6 also; in this case, the control system eliminates the error and stabilizes the process. The control strategy and implementation was developed using numerical simulation (explicit finite element), and the controller implementation was reused directly in the experimental setup without manipulating or scaling the gain factors.
\end{abstract}

Keywords Tube hydro-forming $\cdot$ T-tube $\cdot$ Optimal control $\cdot$ In-process control $\cdot$ Process robustness $\cdot$ Finite element

\section{Introduction}

Feed-forward control strategies are the dominating control strategy applied for the majority of manufacturing processes. As the process layouts were designed, in a time when both modeling capabilities and sensor technology were limited, thus process stability was enforced by conservative process layout. However, the focus is changing, which can be seen by the high number of papers during the last two decades, focusing on both in-process control and part-to-part control schemes applied on metal forming. For an extensive review of the recent developments of process control applied on the metal forming, see [1] and [2].

Typically, the process parameters are very adjustable, and errors might be introduced or initiated early in the process, resulting in process instability in the final stage of the forming operation. The flexible process parameters and difficulties identifying the onset of process instability add to the challenges of process run-in and daily process adjustments.

Benny Endelt

endelt@mp.aau.dk

1 Department of Materials and Production, Aalborg University, Fibigerstraede 16, Aalborg 9220, Denmark
Furthermore, process parameters are in many cases trimmed or adjusted, analyzing the finished part.

A good example is tube hydro-forming, which is a highly flexible process enabling the manufacturing of complex geometries in a single forming operation, where process stability is controlled by two highly adjustable process variables, see Koç and Altan [3]:

- Internal pressure (or the forming pressure) $p(k)$ is responsible for driving the plastic deformation ( $k$ represents the sampling counter or discrete time).

- Axial feeding $s(k)$ is responsible for feeding material to the process. The punch force $F(s)$ drives the punch forward and provides a sealing force preventing leak flow.

Process run-in typically requires several trial and error from an experienced operator where initial punch position, initial pressure, pressure profile, and initial tube length are adjusted/manipulated. The primary objective during run-in is to identify a set of process parameters that are geometrically feasible and robust, i.e., avoiding the three major failure modes, see Koc and Altan [4]:

- Wrinkling — due to insufficient internal pressure or a large axial feeding force. 
- Buckling - due to insufficient internal pressure or a large axial feeding force.

- Bursting - due to a large internal pressure and/or insufficient material feed.

Several approaches have been proposed to improve process feasibility, obtain better quality and more robust processes; starting with rules of thumb/trial and error, various optimization schemes see, e.g., Imaninejad et al. [5], among others Kadkhodayan and Erfani-Moghadam [6] suggest using design of experiments, Aydemir et al. [7] propose adaptive schemes combining a fuzzy control strategy with a finite element-based wrinkling and bursting criterion, Johnson et.al proposed a numerical process control scheme which can forecast the internal pressure and axial feeding Johnson et al. [8]. Common for the strategies is that they try to forecast a suitable or optimal set of process parameters-which will keep the process well within the process window, minimizing the risk of failure.

Manabe et al. propose identifying process parameters, using combination of finite element simulations and a fuzzy control strategy, controlling the contact area between a counter punch and the tube Manabe et al. [9]. The control strategy has also been implemented in an experimental setup for T-tubes Manabe et al. [10], and later a similar approach has also been applied for Y-tubes Nakamori et al. [11].

Groche et al. proposed a volume control strategy, where the process was stabilized by controlling the fluid volume using a classical PID regulator Groche et al. [12].

The present work takes a different approach, where the relation between input and output is modeled applying an explicit finite element model. The gain factors are identified solving a second-order nonlinear optimal control problem, where the dynamic behavior of the system is controlled by two scaling parameters $q_{1}$ and $q_{2}$. The proposed control system is designed using purely a numerical approach where the implementation is debugged and verified off-line.

The control system is tested and evaluated experimentally, where bursting and wrinkling are provoked, manipulating the reference pressure. As a final test, the material is changed, going from an aluminum grade 5049-0 to 6060-T6.

\section{Process and state variables}

The author suggests sampling the filling of the tool cavity by measuring the distance $y(k)$, see Fig. 1 using a mechanical measuring scheme; alternatively, a laser sensor could be applied. We already identified the possible failure modes; however, the system output $y(k)$ should hold sufficient information on two of the failure modes; the relation between the system output $y(k)$ and the failure modes can be described as:

- Under-filling (wrinkling) — the development in $y(k)$ is slower than the reference trajectory $r(k)$. This might introduce wrinkling and is treated by increasing the internal pressure $p(k)$.

- Over-filling (bursting) — the development in $y(k)$ is faster than the reference trajectory $r(k)$. This might introduce bursting and is treated by decreasing the internal pressure $p(k)$.

Note that if the process is sensitive to buckling, more than a single sample point is required.

The process is not governed by time and can be modeled independently of process execution time, defining process progression as a function of axial feeding. Thus, the sampling interval is defined by the punch displacement $s(k)$; in the current setup, the tool filling $y(k)$ is sampled for each axial feeding increment, $\delta s=1.0 \mathrm{~mm}$. Furthermore, time independence allows for some flexibility with respect to process layout, e.g., with respect to initial tube length, speed of axial feeding, punch displacement, and tool filling, as the dynamic behavior, is defined as a function of punch displacement and not time.

Fig. 1 T-tube hydro-forming model illustrating the adjustable process variables punch $F(k)$ and the internal pressure $P(k)$. The punch displacement $s(k)$ is used as a "time" reference for sampling, and distance $y(k)$ is sampled from the process and used to define the state variable $x(k)$

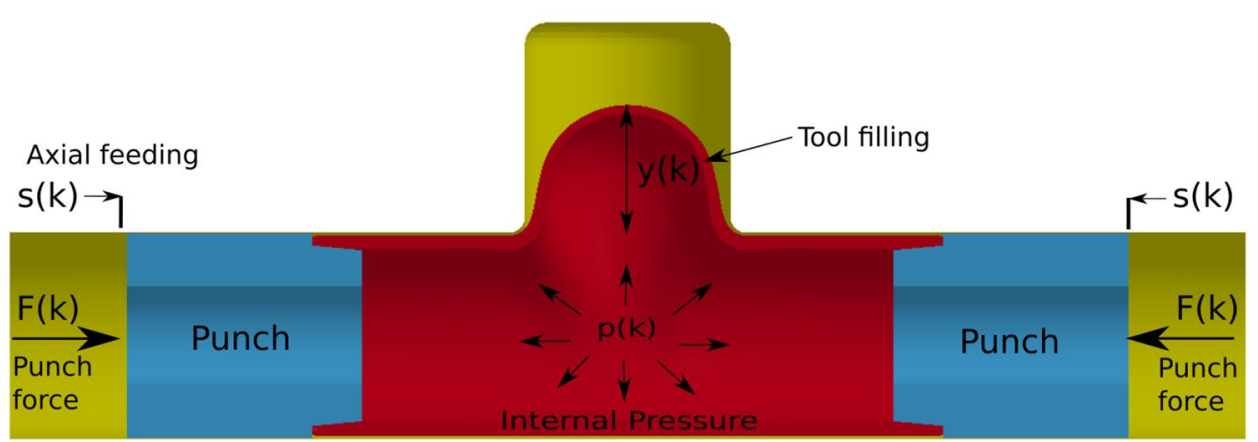


Consequently, the controller design can be implemented directly in the laboratory or production, regardless that both time and mass scaling were applied in the explicit finite element model.

\subsection{Tube and tool dimensions}

The tube material (aluminum 5049-O) was modeled using Voce hardening law $\left(\sigma=\sigma_{0}+\sum \alpha_{i}\left(1-e^{-\beta_{i} \bar{\epsilon}}\right)\right.$, and the material properties were calculated using the uniaxial tensile data reported by Marr et al. [13], see Table 1 .

The following initial tube and tool dimensions were applied:

- Tube dimensions:

- Diameter: $32 \mathrm{~mm}$

- Initial thickness: $1.5 \mathrm{~mm}$

- Length: $160 \mathrm{~mm}$

\section{- Tool dimensions:}

- Nose diameter: $32.0 \mathrm{~mm}$

- T-tube nose height: $30 \mathrm{~mm}$

- Tool filling radius: $5 \mathrm{~mm}$

\subsection{System model}

Tube hydro-forming is a highly nonlinear process with complex relations between axial feeding $\mathrm{s}(\mathrm{k})$, internal pressure $\mathrm{p}(\mathrm{k})$, and operating conditions, e.g., material properties, friction, and tool temperature. Initially, the tube filling is more sensitive to pressure changes, and the pressure sensitivity will reduce as the process progresses; as a result, the relation between $\Delta p(k)=p(k)-p(k-1)$ and $\Delta y(k)=y(k)-y(k-1)$ is nonproportional. The influence of nonproportional relation is reduced using the following pressure update:

$p(k)=w(k)+u(k) w(k)$ for $u \geq-1$

where $w(k)$ represents the reference input; thus, the correction or controller input $u(k)$ is independent of the current internal pressure, i.e., $u=-1$, will reduce the pressure to zero and $u=1$ will double the internal pressure.

Table 1 Voce hardening law-parameters for aluminum alloy 5049-O are calculated based on date reported in Marr et al. [13] and the material parameters for 6060-T6 were reported in Hoang et al. [14]

\begin{tabular}{llllll}
\hline Material & $\sigma_{0}$ & $\alpha_{1}$ & $\alpha_{2}$ & $\beta_{1}$ & $\beta_{2}$ \\
\hline $5049-\mathrm{O}$ & 137.0 & 31.420 & 84.836 & 57.203 & 1.656 \\
$6060-\mathrm{T} 6$ & 170.0 & 64.773 & 34.164 & 13.281 & 2302.815 \\
\hline
\end{tabular}

The objective is to provide an input $u(k)$ which minimizes error $e(k)$, which is defined as the difference between the process output $y(k)$ and a reference trajectory $r(k)$,

$e(k)=y(k)-r(k)$

where the reference trajectory $r(k)$ was produced using the reference input $w(k)$.

Feedback control is based on the simple philosophy; if you cannot see far ahead, then intensify sampling and make small corrections. This is typically implemented using a simple linear update of the input, for the current system. We have a feedback gain matrix $\boldsymbol{K}$ which is used to update the system input $u(k)$ according to:

$$
\begin{aligned}
u(k) & =u(k-1)+\Delta u(k) \\
\Delta u(k) & =\boldsymbol{K}^{T} \boldsymbol{x}(k)
\end{aligned}
$$

The term $\Delta u(k)$ represents the change in the system input from sample to sample, and this correction term $\Delta u(k)$ also represents the controller workload and has a great influence on the dynamic behavior of the system. Further, update formulation Eq. 3 eliminates steady-state errors as the input vector $\mathrm{u}(\mathrm{k})$ is integrated over all previous errors, i.e., the steady-state error is converging to zero.

The gain matrix $\boldsymbol{K}$ was identified by solving an optimal control problem directly based on the nonlinear system model Endelt et al. [15]. The block diagram of the feedback system is given in Fig. 3, where the interaction between LS-Dyna (LS-Dyna version 971) and the feedback loop is illustrated.

\subsection{Finite element model}

The control plant is modeled using a commercial finite element solver LS-DYNA version 971R11. The optimal control problem is solved numerically using finite difference for the sensitivity analysis, i.e., model robustness and numerical stability are very important, why an explicit time integration is applied. The model options are summarized below:

- Due to symmetry, only a quarter of the geometry modeled, see Fig. 4.

- Tool parts are modeled as rigid body, using solid elements for the punches (default element 1) and shell elements for the tool cavity (default element 2), see Fig. 4.

- The tube is modeled using solid element (default element 1) with 3 elements trough the thickness and a total of 16170 elements, see Fig. 4.

- A time step 2E-7 was applied and the simulation time was set at $30 \mathrm{~ms}$, corresponding to a mass scaling ratio of 7 . 
- The material was modeled using Voce hardening, which was chosen to avoid overestimation of the yield stress for large deformation, as the equivalent plastic strain is in the range of 1.0 to 1.5 , dominated by compressive stress. For a comparison of different hardening laws for aluminum see Pham et al. [16], where the Voce hardening law is shown, to slightly underestimate the yield stress when extrapolating, compared to, e.g., Swift and Ghosh hardening laws, which both significantly overestimate the yield stress for larger strains.

- Coulomb friction was assumed using a friction coefficient of 0.1 , and a limiting friction force defines as $F_{\text {lim }}=A_{\text {contact }} \frac{\sigma_{0}}{\sqrt{3}}$ i.e., the friction force is limited by the initial yield stress in pure shear.

The reader should remember, and the system plant is normally modeled using a very simplistic linear model, e.g., a state space formulation. The finite element model shortly described above, should "only" reflect the dynamic behavior of the system, i.e., the system output $y(k)$ when the system input $u(k)$ is manipulated; thus, small modeling errors due to assumptions and numerical errors will have limited impact on the controller design, as the model from a control perspective is very accurate. The main purpose is to produce a model which are numerically stable, relatively fast (CPU-time) and reflect the dynamics of the system. Foremost, the model should provide robust data, which will ensure converge of the optimal control problem defined in the next section.

\subsection{Solving the optimal control problem}

An error is introduced by applying a step input as illustrated in Fig. 2a and b, and the objective is to minimize an object function $f(\boldsymbol{K})$. The object function contains two termsminimizing both the filling error $e(k)$ and the change in the controller input $\Delta u(k)$ :
$f(\boldsymbol{K})=\frac{1}{2} \sum_{k=1}^{m} q_{1} e^{2}(k)+\frac{1}{2} \sum_{k=1}^{m} q_{2} \Delta u^{2}(k)$

where $m$ represents the number of samples. The object function is equivalent to the objective function used in optimal control in the linear case. The weighting coefficients $q_{1}$ and $q_{2}$ controlled the dynamic behavior and stability of the system, where $q_{1}$ controls the impact of the filling error and penalty factor, and $q_{2}$ controls the system damping.

\section{Identification of gain factors}

The nonlinear optimal control problem is solved using a Gauss-Newton formulation where the step size is regulated using a trust-region scheme and the nonlinear object function $f(\boldsymbol{K})$ is evaluated using an explicit finite element model. Minimizing $f(\boldsymbol{K})$ for a system subject to a systematic error (a step input):

$p^{i d}(k)=w(k)+v(k) w(k)$

where $w(k)$ represents the reference internal pressure, see Fig. 2b. Figure 2a plots the disturbance v(k), using a step input of -0.4 , applied for the first half of the tube-forming simulation and set to zero for the second half of the forming operation. This step input will stress the controller twice, i.e., a smooth increasing error development during the first half of the simulation, followed by a step-input sample 20 $(k=20)$, see Fig. $2 b$ and a. The optimal control problem is solved using a fixed error penalty $q_{1}=1$ and different values of input penalty factor $q_{2}$ (increasing the system damping). The problem is typically solved with four to seven iterations, involving 12 to 23 finite element simulations.

Figure 5 shows a clear correlation between the system damping and the penalty factor $q_{2}$, i.e., $q_{2}=1$ results in a system with an overshot oscillating input (from sample 20

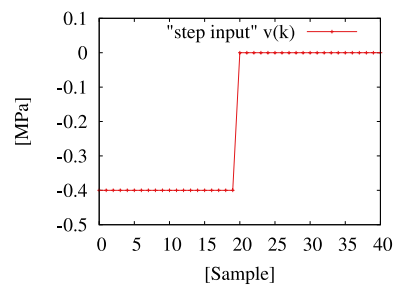

(a) Controller disturbance $v(k)$.

Fig. 2 Reference internal pressure $p(k)$ and axial feeding $s(k)$ trajectories using the reference material parameters, Table 1

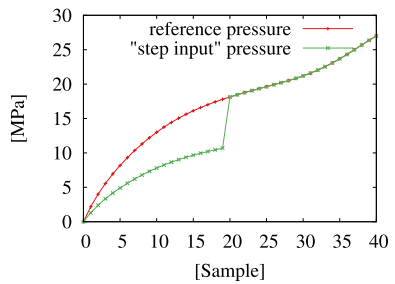

(b) Reference internal pressure and the "step input" pres- $s(k)$ sure used during identification of gain factors.

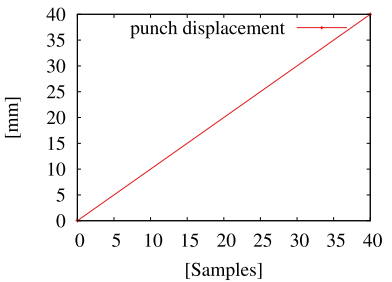

(c) Axial feeding trajectory $(k)$. rajectory.

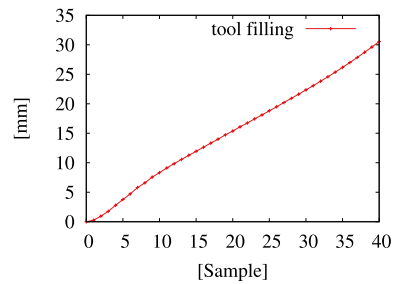

(d) Reference tool filling $y(k)$ 
Fig. 3 In the block diagram of pressure $p(k)$ is a function of both the reference pressure and the input $u(k)$ i.e., $\mathrm{u}=-1$, will reduce the pressure to zero, and $\mathrm{u}=1$ will double the pressure the control loop, note that the

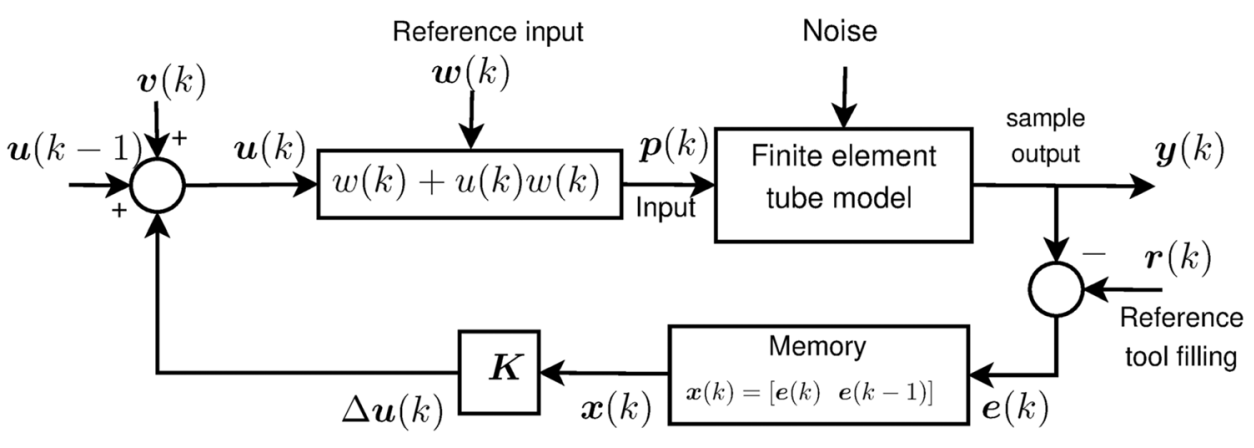

The main components in the experimental setup:

forward) where $q_{2}=10$ produces a well-damped input and output signal again from sample 20 and forward.

Selecting gain factor is depending on the desired dynamical behavior of the control system and is a compromise between settling time and tendency to overshoot or oscillate. Based on the system responses Fig. 5c the gain factor identified for $q_{2}=2$ represents a fair compromise between system damping and settling time, see Table 2.

\section{Experimental setup}

Figure 6 shows the experimental setup, where the tube forming unit is mounted in a single-action hydraulic press (capacity 120 ton). To keep the system as open and flexible as possible, the control system is implemented in Java, and the communication between the computer and the tube forming unit is established using a universal serial bus (USB). Furthermore, the controller developed during the numerical tests is reused for the experimental setup, i.e., the implementation is verified and debugged in a virtual setup, enabling full control and repeatability without; time delays, sensor fallout, sampling noise, etc. Only the interface communicating with the external components is changed for the physical setup.

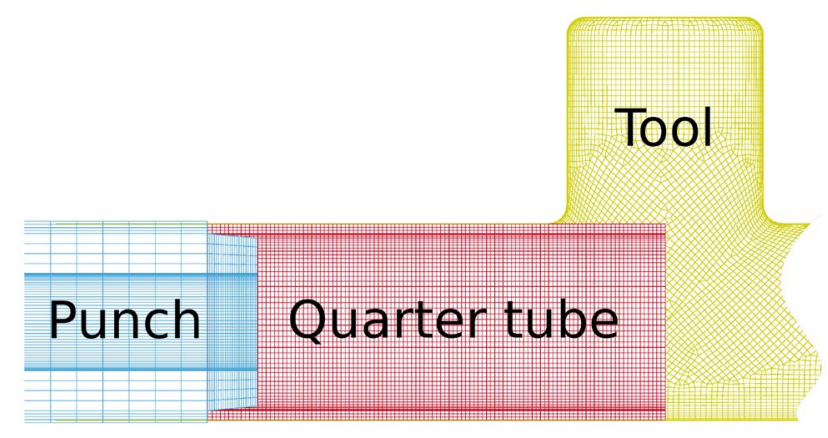

Fig. 4 Finite element mesh, due to symmetry assumptions only a quarter of the tube is modeled
- The tool is mounted in a 120-ton single-action hydraulic press.

- Hydraulic punches 0-200 $\mathrm{kN}$ feeding force, punch speed $0-20[\mathrm{~mm} / \mathrm{sec}]$.

- The punch displacement is individually controlled using two proportional valves.

- Both punches have built-in position sensors.

- High-pressure hydraulic (internal pressure)—maximum pressure $700[\mathrm{Bar}]$ and $1.3[1 / \mathrm{min}]$.

- The forming pressure is regulated using a proportional valve, pressure range 0-700[Bar], and max flow 25[1/ $\min ]$.

- Hydraulic fluid and lubrication: Shell Tellus S3 M. dled using two modules from LucidControl, a 4-channel analog input (4-20mA) module, and a 4-channel output module (4-20mA), both with a USB interface.

The pressure profile, initial tube length $(160 \mathrm{~mm})$, and axial feeding $(47 \mathrm{~mm}$ ) are based on trial and error (using the experience from the simulations). Figure 7 shows the reference geometry, pressure $p(k)$, and tool filling profile $r(k)$.

\section{Experimental sesults}

The performance of the control system is evaluated by provoking the two common error modes associated with tube hydro-forming:

- Bursting: The error is provoked by doubling the reference pressure $\mathrm{w}(\mathrm{k})(p(k)=w(k)+w(k) v(k)$ where $v(k)=1$ ), see Fig. 8b. Figure 8a llustrates the resulting filling error, and Fig. 8c shows the applied internal pressure.
- The controller is implemented in java and I/O is han- 


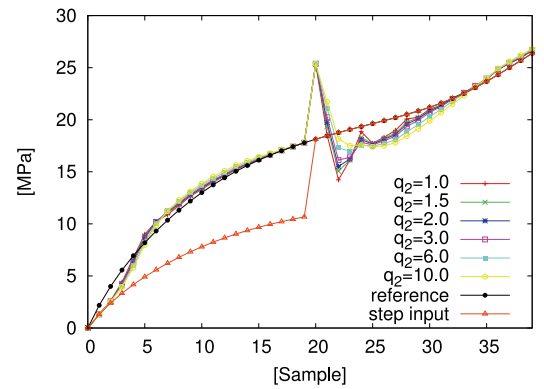

(a) Change in controller input $\Delta u(k)=$ $\boldsymbol{K} \boldsymbol{x}(k)$

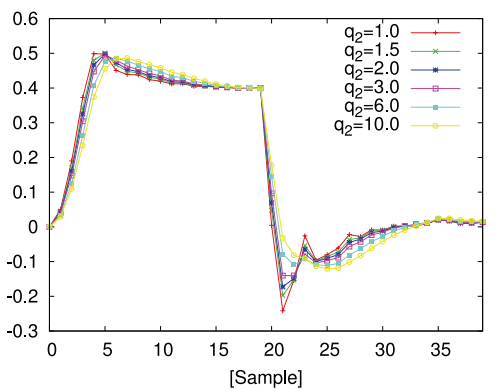

(b) Controller input $u(k+1)=u(k)+$ $\Delta u(k)$

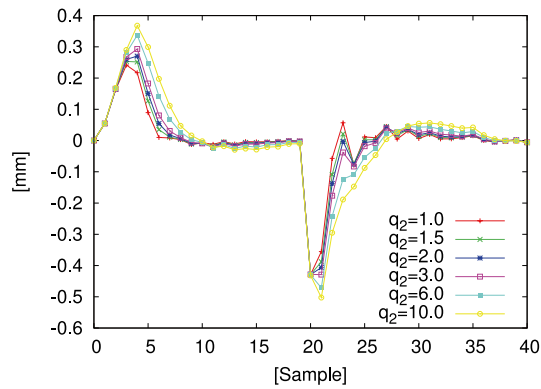

(c) Tool filling error.

Fig. 5 Tool filling error and internal pressure — note the correlation between system damping and input penalty factor $q_{2}$

- Feed-forward: The filling error (over-filling) is developing rapidly within the first five samples and almost instantaneously bursting — at a punch displacement of only $10 \mathrm{~mm}$, see Fig. 8d.

- Feedback: As expected, the control system reduces the internal pressure $p(k)$ and stabilizes the process, Fig. 8. Note, input $u(k)$ converges to -1 , eliminating disturbance $v(k)=1$, see Fig. 8b.

- Wrinkling: The error is provoked by reducing the reference pressure $\mathrm{w}(\mathrm{k})$ using half the pressure $(p(k)=w(k)+w(k) v(k)$ where $v(k)=-0.5)$, see Fig. 9b. Figure 9a illustrates the resulting filling error, and Fig. 9c shows the applied internal pressure.

- Feed-forward: The filling error is again developing rapidly, as expected under-filling the tool cavity. Figure 9a shows a steady increasing error from sample 5 and forward (a punch displacement of $5 \mathrm{~mm}$ ) —indicating limited filling and significant wrinkling, see Fig. 9d.

Table 2 Feedback gain factors for the control system for different values of the penalty factor $\boldsymbol{q}_{2}$ (fixed value $q_{1}=1.0$ ). The final value of the object function is summarized in the last tree columns $f_{q 1}=\frac{1}{2} \sum_{k=1}^{m} q_{1} * e^{2}(k)$ and $f_{q 2}=\frac{1}{2} \sum_{k=1}^{m} q_{2} \Delta u^{2}(k)$ and summation $f(\boldsymbol{K}) \stackrel{2}{=} f_{q_{1}}+f_{q_{2}}$, see Eq. 4

\begin{tabular}{llllll}
\hline \multicolumn{6}{l}{ Initial gain factors $k_{1}=0.75$ and $k_{2}=-0.5$} \\
\hline$q_{2}$ & $k_{1}$ & $k_{2}$ & $f_{q_{1}}$ & $f_{q_{2}}$ & $f(\boldsymbol{K})$ \\
\hline 1.0 & 1.2693 & -0.7159 & 0.1967 & 0.1616 & 0.3583 \\
1.5 & 1.1327 & -0.6445 & 0.2295 & 0.2017 & 0.4312 \\
2.0 & $\mathbf{1 . 0 6 3 4}$ & $\mathbf{- 0 . 6 1 2 1}$ & 0.2526 & 0.2444 & 0.4970 \\
3.0 & 0.9770 & -0.5670 & 0.2873 & 0.3244 & 0.6117 \\
6.0 & 0.8225 & -0.4831 & 0.3761 & 0.5200 & 0.8962 \\
10.0 & 0.7150 & -0.4204 & 0.4706 & 0.7455 & 1.2161 \\
\hline
\end{tabular}

- Feedback: The control system increases the internal pressure and eliminates wrinkling, see Fig. 9e. Furthermore, the controller input $u(k)$ converges to 0.5 (doubling the input pressure) within the first 10 samples, i.e., the disturbance $\mathrm{v}(\mathrm{k})=-0.5$ is eliminated, see Fig. 9 b.
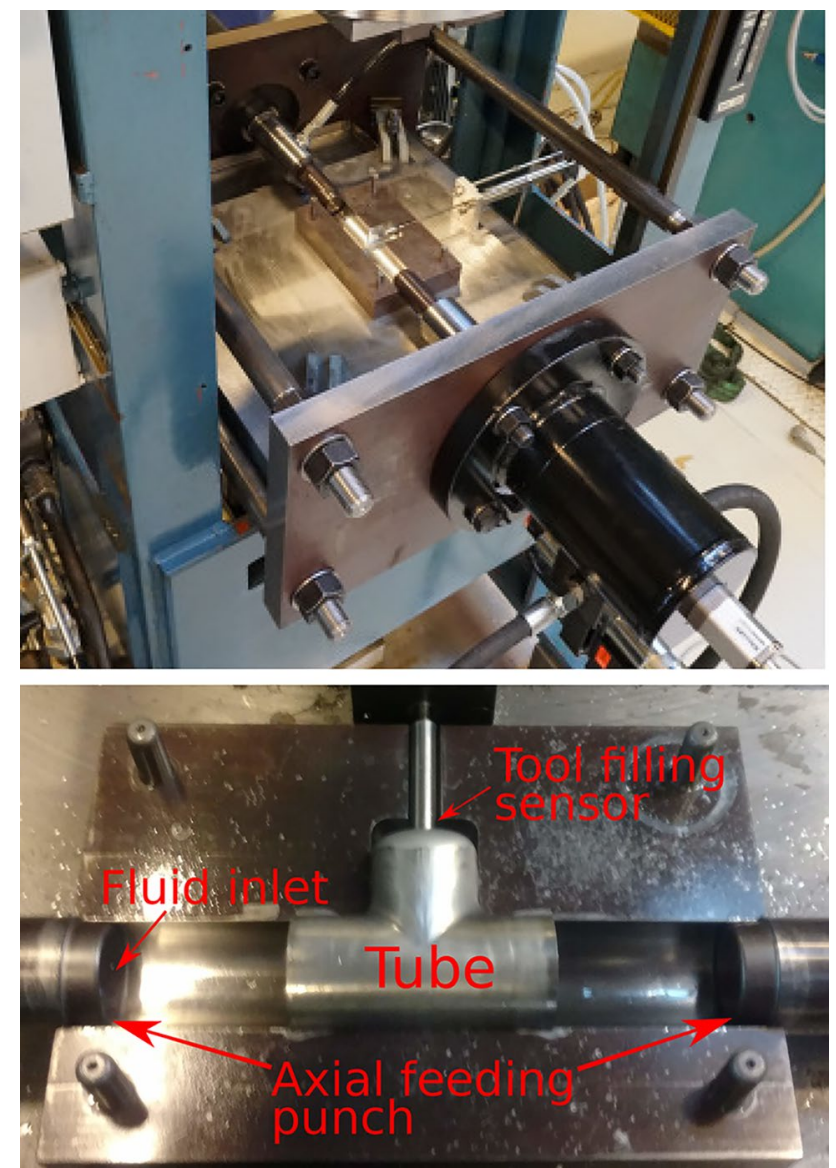

Fig. 6 Experimental setup 


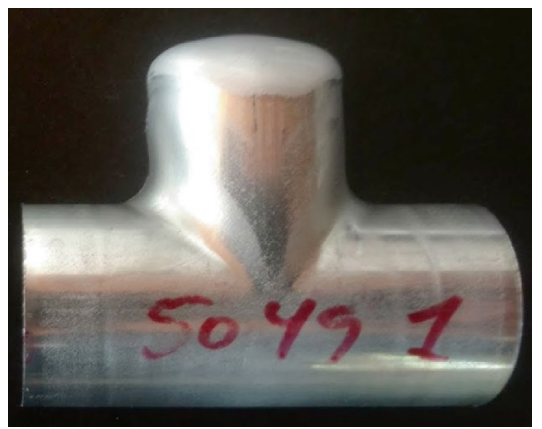

(a) Reference T-tube

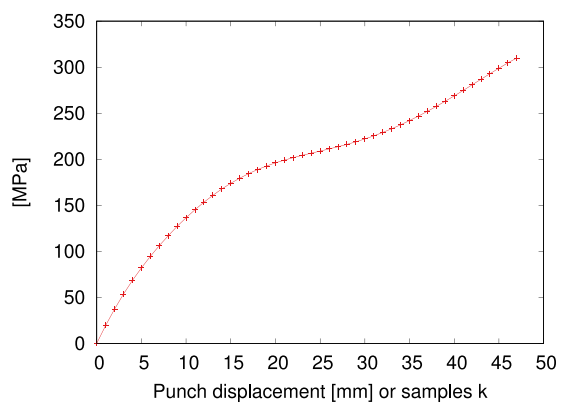

(b) Reference pressure.

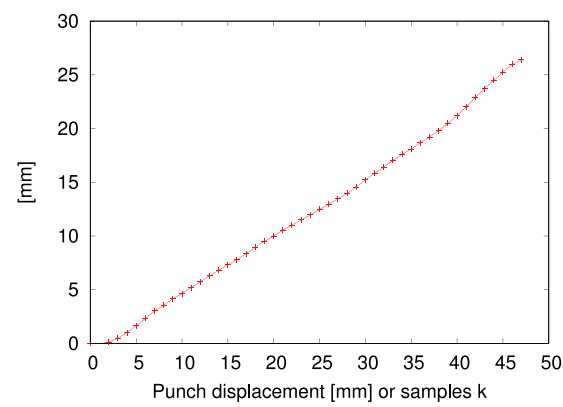

(c) Reference tool filling.

Fig. 7 Reference part and process parameters (material 5049-O)

- Step input: The step input is applied using an pressure profile $p(k)=w(k)+w(k) v(k)$ where $v(k)=-0.3$ the first 19 samples and then return to the reference pressure $p(k)=w(k)$, see Fig. 10b. Reusing the disturbance used during the identification of gain factors.

- Feed-forward: The reduced initial pressure will initiate wrinkling during the first 19 samples, which is not eliminated by the pressure returns to the reference pressure, see Fig. 10d.

- Feedback: The pressure $p(k)$ returns to the reference pressure $w(x)$ from sample 20 and forward. The dynamic behavior of the system can clearly be observed from sample 20 and forward, where the input $u(k)$ oscillates and stabilizes at zero from sample 32 and forward. Note, the real system has a more dynamic response from sample 20 and for-

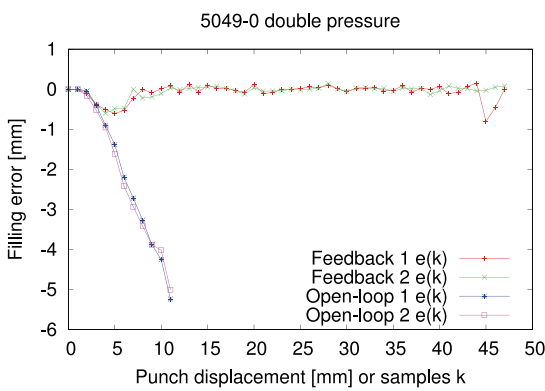

(a) Filling error $\mathrm{e}(\mathrm{k})$.

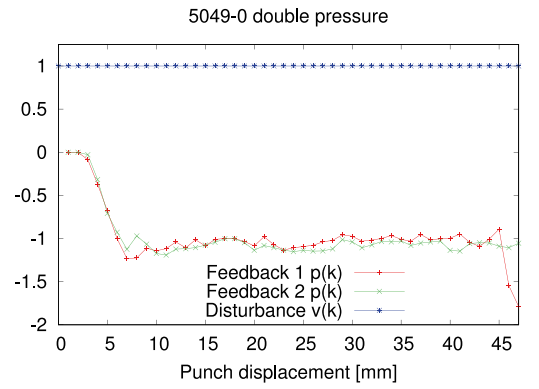

(b) Controller input $\mathrm{u}(\mathrm{k})$ converging to $u(k)=-v(k)$ eliminating the effect of the disturbance $v(k)$.

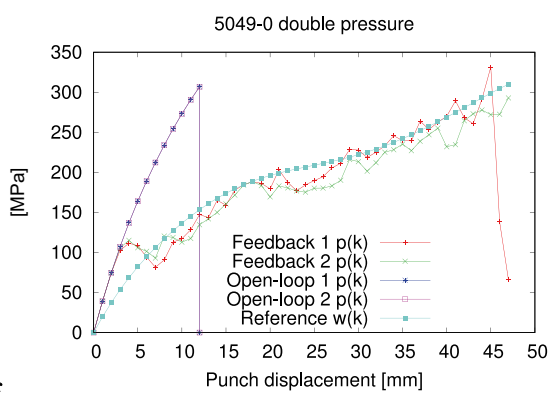

(c) Input pressure $\mathrm{p}(\mathrm{k})$.

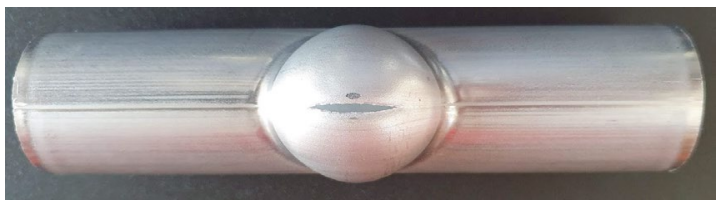

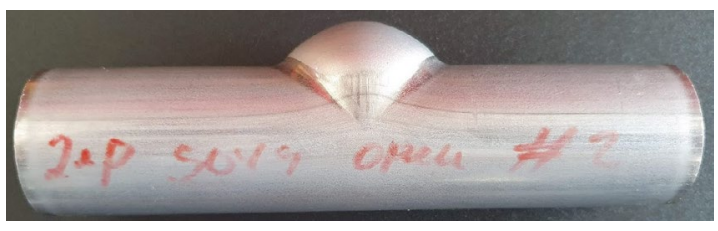

(d) Open-loop.

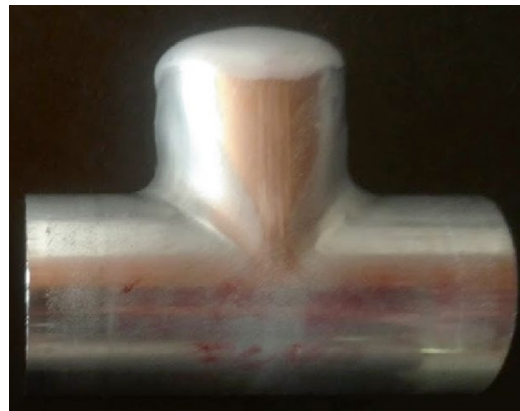

(e) Feedback.

Fig. 8 Results from the bursting experiment (double the forming pressure), where the feedback system stabilizes the process by reducing the pressure (material 5049-O) 


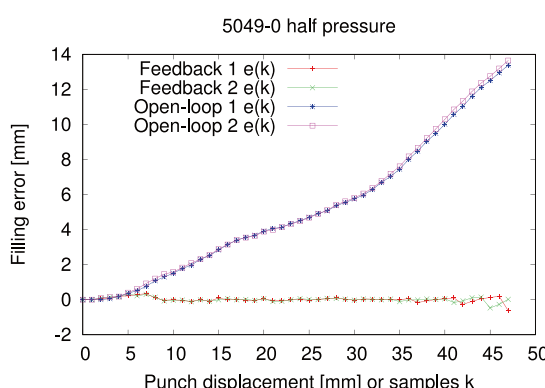

(a) Filling error $\mathrm{e}(\mathrm{k})$.

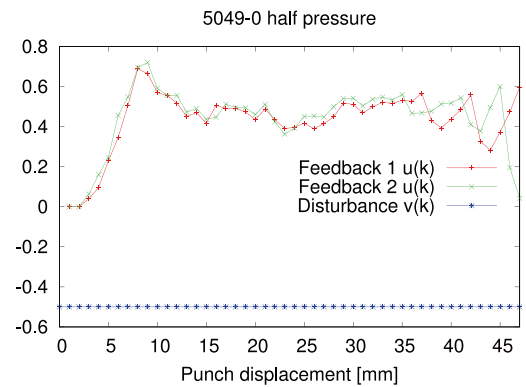

(b) Controller input $\mathrm{u}(\mathrm{k})$ converging to $u(k)=-v(k)$ eliminating the effect of the disturbance $v(k)$.

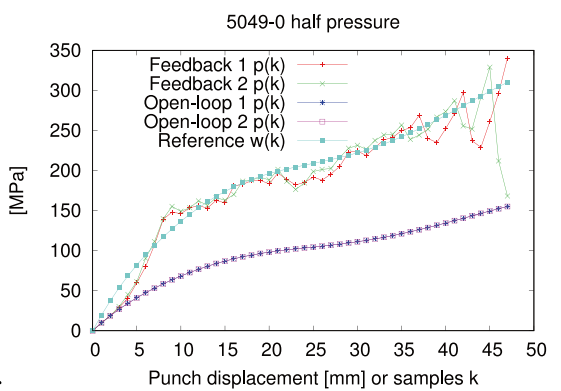

(c) Input pressure $\mathrm{p}(\mathrm{k})$.

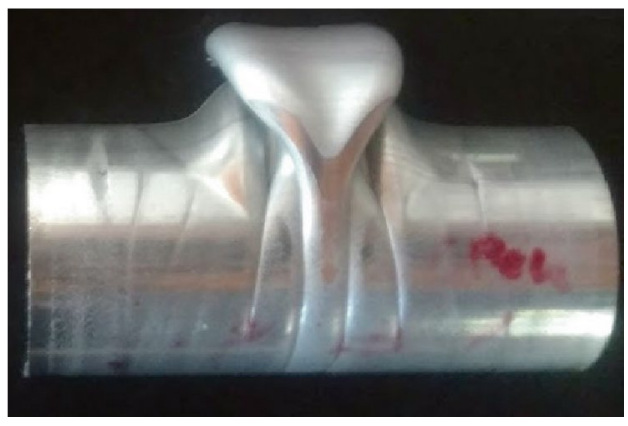

(d) Open-loop.

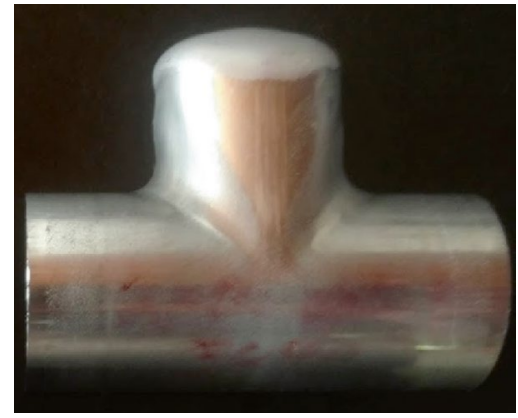

(e) Feedback.

Fig. 9 Wrinkling error using a low forming pressure, the feedback system stabilizes the process by increasing the internal pressure (material 5049-O)

ward compared to the numerical model Fig. 5c, where the experiment shows both larger errors and settling time. The difference between model response and experiments could be related to the material model overestimating the yield stress. Furthermore, the friction was modeled using Coulomb friction

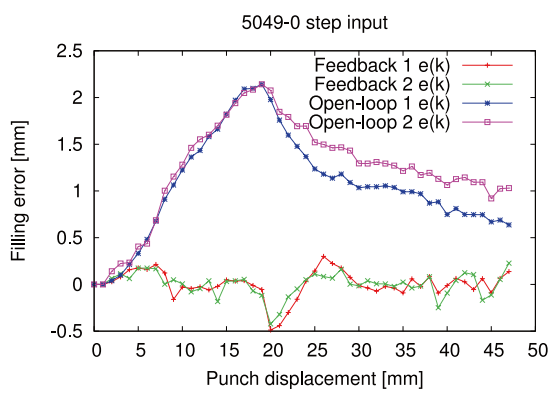

(a) Filling error $\mathrm{e}(\mathrm{k})$.

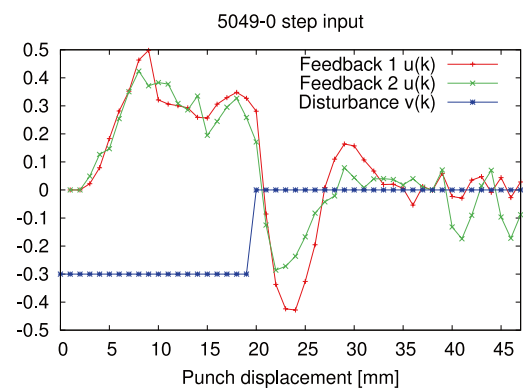

(b) Controller input $\mathrm{u}(\mathrm{k})$.

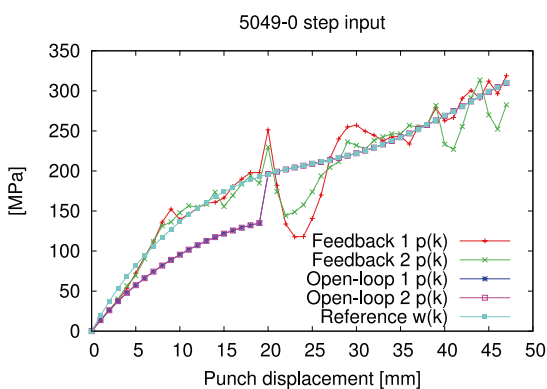

(c) Input pressure $\mathrm{p}(\mathrm{k})$.

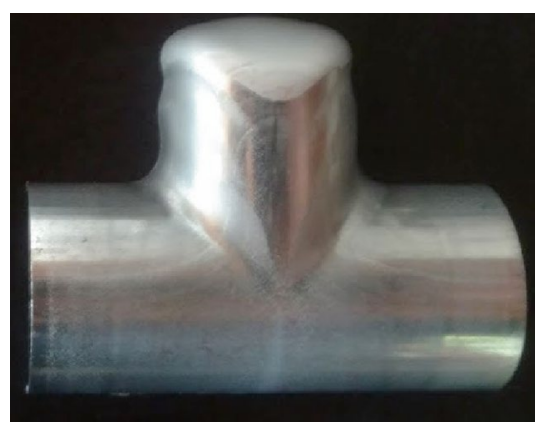

(d) Open-loop.

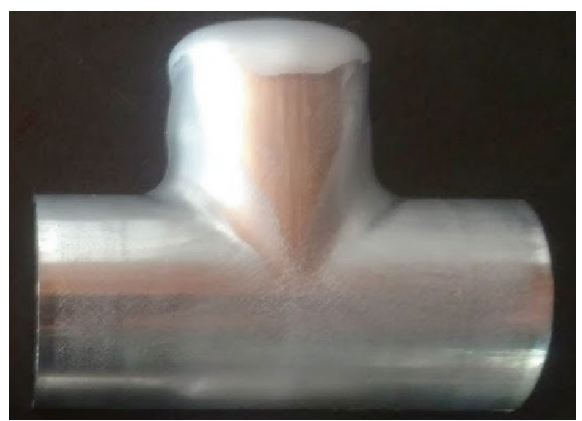

(e) Feedback.

Fig. 10 Step input response- the dynamic behavior of the system can clearly be observed from sample 20 and forward where the input $\mathrm{u}(\mathrm{k})$ oscillates and stabilizes at zero from sample 32 and forward (material 5049-O) 


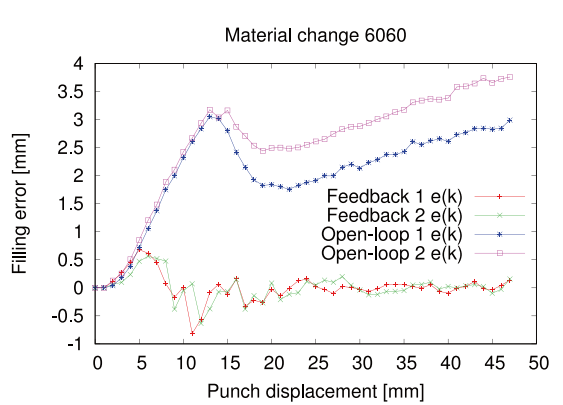

(a) Filling error $\mathrm{e}(\mathrm{k})$.

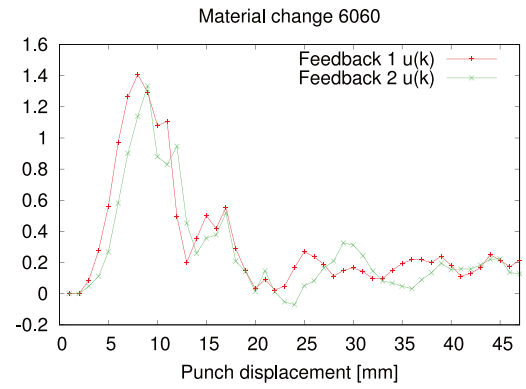

(b) Controller input $\mathrm{u}(\mathrm{k})$.

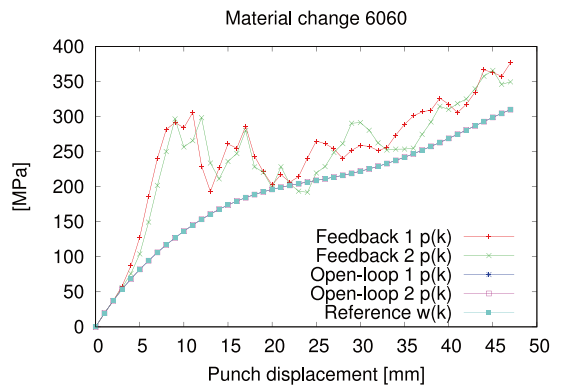

(c) Input pressure $\mathrm{p}(\mathrm{k})$.

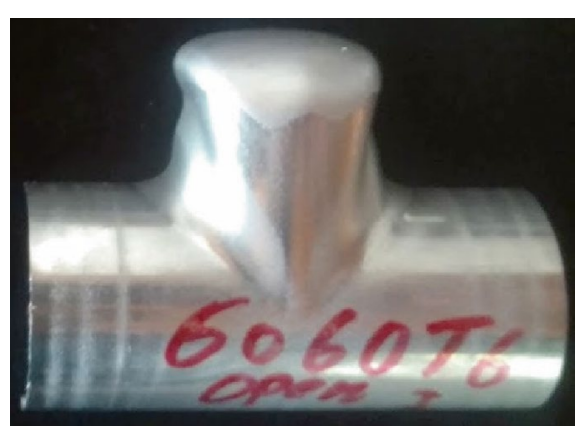

(d) Open-loop.

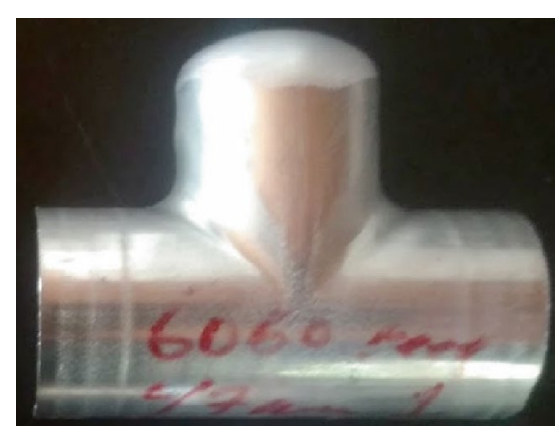

(e) Feedback.

Fig. 11 New material is introduced 6060-T6, and the feedback system stabilizes the process by increasing the internal pressure

( $\mu=0.1$, which also contributes to the observed differences. However, the deviation between modeled and experimental responses does not affect the system stability.

The experimental tests above clearly illustrate that the control system has a high capability of handling errors which are commonly seen in tube hydro-forming, i.e., bursting and wrinkling. However, the errors were provoked by manipulating the input signal or reference pressure, i.e., the dynamic behavior of the system is not changed during the tests. In the final test of the control system, the tube material is changed going from 5049-O to 6060-T6, see the difference in material parameters Table 1 and hardening curves Fig. 12.

- New material 6060-T6: Changing material is the final test of the control system. As the material manipulates, the overall system response and a more unpredictable and dynamic system response might be expected.

- Feed-forward The material change causes under-filling and wrinkling, see Fig. 11a and d. Note the rapid rising error during the first 13-14 samples. From samples 13 to 20 , the error is reduced, and finally, steady increases throughout the remaining forming operation.
- Feedback The control system efficiently eliminates the under-filling error, by correcting the pressure by a factor of 1.4 during the first 10 samples, see Fig. 11b. As for the feed-forward case, the effect of the material change decreases from samples 13 to 20 , and the controller correction is in a steady state from samples 25 and forward.

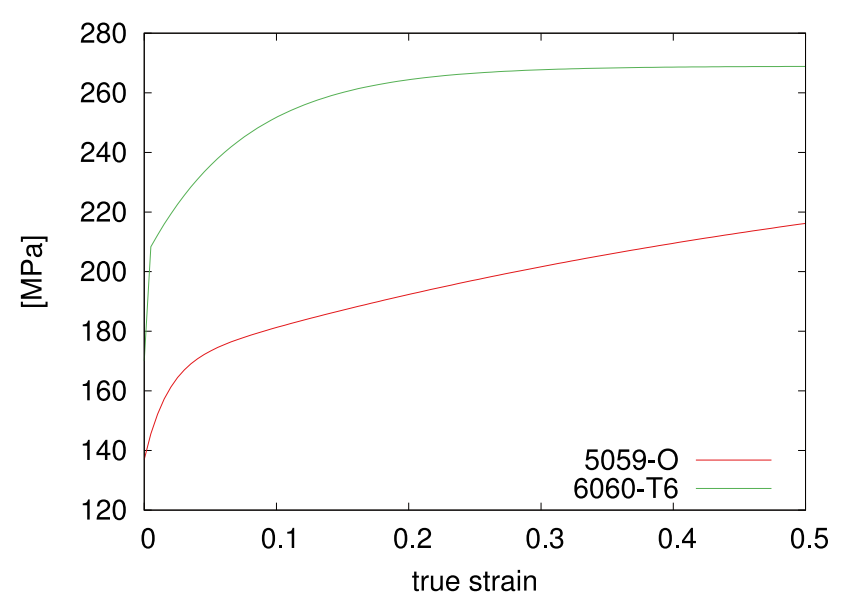

Fig. 12 Voce hardening law plotted for aluminum alloy 5049-O and 6060-T6. The material parameters are listed in Table 1 


\section{Conclusion}

A novel framework for modeling and designing control systems for highly nonlinear processes was proposed, where the controller input was defined using successive samples in the current case the filling of the tube cavity. The system plant was modeled using a nonlinear finite element model, and the gain factors were identified, solving a nonlinear optimal control problem, using a Gauss-Newton nonlinear least-squares solver.

The control problem is nontrivial as wrinkling or localization (bursting) will develop rapidly and, in most cases, are irreversible. Thus, the control system should react fast and without extensive overshoot, as an overshoot, may initiate irreversible wrinkling or localization.

The control system was verified experimentally, using four different errors scenarios. Where error was provoked, manipulating the input signal in the first three cases, the control system successfully eliminated both wrinkling and bursting. Finally, the material was changed, going from 5049-O to 6060-T6; again, the control system was able to stabilize the process.

The control strategy and implementation were developed using numerical simulations. The identified gain factors and the implemented controller were reused directly in the experimental setup, without gain factor manipulation or scaling. Only a new interface was needed, moving from communicating with LS-Dyna to the experimental setup, i.e., proportional valves, pressure, and inductive sensors, etc.

The meteorology proved stable and cost-efficient from a manufacturing point of view, as the controller design was based on numerical simulation, i.e., experiments were only used to verify the numerical findings.

\section{Future research}

The presented in-process control system corrects errors during the processing of the part. However, the limitation of this approach is that we do not pick up information from part to part, e.g., if a new material batch is introduced, the controller will, part after part, correct the same errors. Thus, an additional control loop which, pass process information from part to part, would enhance the performance of the control system. The basic idea is to update the reference pressure trajectory $w(k)$ based on historical process information; this control scheme is known as iterative learning control (ILC), see Endelt [17].

Author contributions Not applicable-Single Author.
Funding information The author would like to thank the following private foundation for contributing to the research, P.A. Fiskers Fond, Det Obelske Familiefond, Henry og Mary Skov, Tranes Fond, Ole Kirk's Fond, and Fabrikant Mads Clausens Fond, and for providing funding for the experimental equipment.

Data availability statement Not applicable.

Code availability A combination of commercial software LS-DYNA and custom research code is written in Java; on request, the author will make the code available.

\section{Declarations}

Conflicts of interest/Competing interests There is no conflict of interest as the presented work can be regarded as free research, driven by the author's own ideas and research interest.

Ethics approval Not applicable.

Consent to participate Not applicable.

Consent for publication Not applicable.

Open Access This article is licensed under a Creative Commons Attribution 4.0 International License, which permits use, sharing, adaptation, distribution and reproduction in any medium or format, as long as you give appropriate credit to the original author(s) and the source, provide a link to the Creative Commons licence, and indicate if changes were made. The images or other third party material in this article are included in the article's Creative Commons licence, unless indicated otherwise in a credit line to the material. If material is not included in the article's Creative Commons licence and your intended use is not permitted by statutory regulation or exceeds the permitted use, you will need to obtain permission directly from the copyright holder. To view a copy of this licence, visit http://creativecommons.org/licenses/by/4.0/.

\section{References}

1. Polyblank JA, Allwood JM, Duncan SR (2014) Closed-loop control of product properties in metal forming: A review and prospectus. J Mat Process Technol 214(11):2333-2348. https://doi.org/10. 1016/j.jmatprotec.2014.04.014

2. Allwood J, Duncan S, Cao J, Groche P, Hirt G, Kinsey B, Kuboki T, Liewald M, Sterzing A, Tekkaya A (2016) Closed-loop control of product properties in metal forming. CIRP Annals 65(2):573596. https://doi.org/10.1016/j.cirp.2016.06.002

3. Koç SM, Altan T (2002) Prediction of forming limits and parameters in the tube hydroforming process. Int J Mach Tools Manuf 42(1):123-138. https://doi.org/10.1016/S0890-6955(01)00048-7

4. Koc M, Altan T (2001) An overall review of the tube hydroforming (thf) technology. J Mat Process Technol 108(3):384-393

5. Imaninejad M, Subhash G, Loukus A (2005) Loading path optimization of tube hydroforming process. Int J Mach Tools Manuf 45(12):1504-1514. https://doi.org/10.1016/j.ijmachtools.2005.01. 029

6. Kadkhodayan M, Erfani-Moghadam A (2012) An investigation of the optimal load paths for the hydroforming of $t$-shaped tubes. Int $J$ Adv Manuf Technol 1-13

7. Aydemir A, De Vree J, Brekelmans W, Geers M, Sillekens W, Werkhoven R (2005) An adaptive simulation approach designed for tube hydroforming processes. J Mater Process Technol 159(3):303-310 
8. Johnson K, Nguyen BN, Davies R, Grant G, Khaleel M (2004) A numerical process control method for circular-tube hydroforming prediction. Int J Plast 20(6):1111-1137. https://doi.org/10.1016/j. ijplas.2003.10.006. URL https://www.sciencedirect.com/science/ article/pii/S0749641903001451, recent Advances in Multiscale Modeling of Plasticity

9. Manabe K, Suetake M, Koyama H, Yang M (2006) Hydroforming process optimization of aluminum alloy tube using intelligent control technique. Int J Mach Tool Manuf 46(11):1207-1211

10. Manabe K, Chen X, Kobayashi D, Tada K (2014) Development of in-process fuzzy control system for t-shape tube hydroforming. Procedia Engineering 81:2518-2523, https://doi.org/10.1016/j. proeng.2014.10.360, 11th International Conference on Technology of Plasticity, ICTP (2014) 19-24 October 2014. Nagoya Congress Center, Nagoya, Japan

11. Nakamori T, Shukuno K, Manabe K (2017) In-process controlled $\mathrm{y}$-shape tube hydroforming with high accurate built-in sensors. Proc Eng 184:43-49. https://doi.org/10.1016/j.proeng.2017.04. 069. Adv Mat Proc Technologs Conf

12. Groche P, Steinheimer R, Schmoeckel D (2003) Process stability in the tube hydroforming process. CIRP Annals 52(1):229-232. https://doi.org/10.1016/S0007-8506(07)60572-1

13. Marr T, Freudenberger J, Maier V, Höppel HW, Göken M, Schultz L (2014) The strengthening effect of phase boundaries in a severely plastically deformed ti-al composite wire. Metals
4(1):37-54. https://doi.org/10.3390/met4010037. URL https:// www.mdpi.com/2075-4701/4/1/37

14. Hoang NH, Porcaro R, Langseth M, Hanssen AG (2010) Selfpiercing riveting connections using aluminium rivets. Int $\mathrm{J}$ Solids Struct 47(3):427-439. https://doi.org/10.1016/j.ijsolstr.2009.10. 009

15. Endelt B, Tommerup S, Danckert J (2013) A novel feedback control system - controlling the material flow in deep drawing using distributed blank-holder force. J Mater Process Technol 213(1):36-50. https://doi.org/10.1016/j.jmatprotec.2012.08.003

16. Pham QT, Lee BH, Park KC, Kim YS (2018) Influence of the post-necking prediction of hardening law on the theoretical forming limit curve of aluminium sheets. Int J Mech Sci 140:521-536. https://doi.org/10.1016/j.ijmecsci.2018.02.040. URL https://www. sciencedirect.com/science/article/pii/S0020740317316235

17. Endelt B (2017) Design strategy for optimal iterative learning control applied on a deep drawing process: Recognising that stamping and deep-drawing operations are repetitive processeswhich can learn and improve based on process history. Int $\mathrm{J}$ Adv Manuf Technol 88(1-4):3-18. https://doi.org/10.1007/ s00170-016-8501-z

Publisher's Note Springer Nature remains neutral with regard to jurisdictional claims in published maps and institutional affiliations. 